\title{
CAUSAL CONFIGURATIONS OF HOMOGENEOUS ENERGY DENSITY IN GENERAL RELATIVITY
}

\author{
P. S. NEGI \\ Department of Physics, Kumaun University \\ Nainital, 263 002, India \\ negi@upso.ernet.in
}

Received Day Month Year

\begin{abstract}
If the causality condition [the speed of sound always remains less than that of light in vacuum, i. e., $v \leq c=1$ ] is imposed on the spheres of homogeneous energy density, the 'ratio of the specific heats', $\gamma \leq 2.59457$, constraints the compaction parameter, $u[\equiv$ $(M / a)$, mass to size ratio in geometrized units] of the dynamically stable configurations $\leq 0.34056$ [corresponding to a surface redshift $\left(z_{a}\right) \leq 0.771$ ]. Apparently, The maximum value of $u$ obtained in this manner belongs to an absolute upper bound, and gives: (i) The maximum value for static neutron star masses as $5.4 M_{\odot}$, if we substitute the density at the surface of the configuration equal to the average nuclear density, $E=2 \times 10^{14} \mathrm{~g} \mathrm{~cm}^{-3}$ [e.g. Nature, 259, 377 (1976)]. (ii) However, if the density of the static configuration is constrained to the value $1.072 \times 10^{14} \mathrm{~g} \mathrm{~cm}^{-3}$, by imposing the empirical result that the minimum rotation period of the fastest rotating pulsar known to date, PSR $1937+$ 21 , is $1.558 \mathrm{~ms}$, the maximum mass value for static neutron stars exceed upto $7.4 M_{\odot}$. These masses have important implications for the massive compact objects like Cyg X-1, Cyg XR-1, and LMC-X3 etc., which may not, necessarily, represent black holes. (iii) The minimum rotation periods for a static $1.442 M_{\odot}$ neutron star to be $0.3041 \mathrm{~ms}$.

(iv) A suitable stable model of ultra-compact objects $[u>(1 / 3)]$ which has important astrophysical significance.
\end{abstract}

Keywords: neutron stars; pulsars; dynamical stability.

\section{Introduction}

Incompressible fluid spheres of uniform energy density $E$ in General Relativity were first discussed by Schwarzschild. ${ }^{1}$ The importance of this solution in General Relativistic stellar structures is apparent, because it gives an absolute upper limit on compaction parameter, $u(\equiv M / a$, mass to size ratio of entire configuration in geometrized units $) \leq(4 / 9)$ for any regular static solution in hydrostatic equilibrium. ${ }^{2}$

Chandrasekhar ${ }^{3,4}$ discussed the condition of hydrostatic equilibrium under the small adiabatic perturbations, and showed that for each value of the compaction parameter, corresponding to the compressible homogeneous spheres, there exists a critical (minimum) value of the "ratio of specific heats", $\gamma\left(=\gamma_{\text {crit }}\right)$ such that for $\gamma<\gamma_{c r i t}$, the configuration becomes dynamically unstable. For the limiting case of the compaction parameter approaching the Schwarzschild limit $(u=4 / 9), \gamma$ 
becomes infinity.

For dynamically stable superdense objects like neutron stars one may expect a finite value of $\gamma$. However, for such objects the equations of state are not well known [empirically] beyond the density $\cong 10^{14} \mathrm{~g} \mathrm{~cm}^{-3},{ }^{5}$ and one can only extrapolate the equations of state (available in the literature) ${ }^{6}$ far beyond this density. As a way out, one can impose some restrictions upon the known physical quantities, such that, the speed of sound inside the configuration,

$$
v \equiv \sqrt{(\partial P / \partial E)_{s}}
$$

(Where $P$ is the pressure, $E$ is the energy density and $s$ stands for specific entropy) does not exceed the speed of light in vacuum, i.e., $v \leq c=1$ (in geometrized units), and obtain an upper bound on stable neutron star masses. ${ }^{7-9}$

In the present paper, we have obtained an upper bound on compaction parameter $(u \leq 0.34056$ corresponding to a surface redshift of 0.771$)$ for the compressible homogeneous spheres, ${ }^{3,4}$ by imposing constraint on the "ratio of specific heats", $\gamma[\leq 2.59457)]$, compatible with causality $(v \leq 1)$ and dynamical stability. This value of the compaction parameter is an absolute maximum because, for an assigned value of $\gamma$, the maximum compactness would correspond to a compressible uniform density sphere, and can be used to obtain an upper bound on neutron star masses, as well as the minimum rotation period of a $1.442 M_{\odot}$ neutron star (the maximum mass of the neutron star accurately known at present). ${ }^{10}$

\section{Equations Governing Radial Pulsations and Limits on Compaction Parameter Imposed by Causality and Ratio of the Specific Heats}

Chandrasekhar ${ }^{3,4}$ discussed the dynamical stability of fluid spheres with respect to small radial adiabatic oscillations on the basis of Einstein's field equations for a metric of the form

$$
d s^{2}=e^{\nu} d t^{2}-e^{\lambda} d r^{2}-r^{2} d \theta^{2}-r^{2} \sin ^{2} \theta d \phi^{2},
$$

where $\nu$ and $\lambda$ are functions of $r$ and $t$. By considering the general time-dependent field equations appropriate for the metric given by Eq. (1), and letting the quantity $\xi(r)$ represent the amplitude of the 'Lagrangian displacement' from the equilibrium position, namely, $\xi(r, t)=\xi(r) e^{-i \sigma t}$, where $\sigma$ is the angular frequency of the pulsation, the variational base for determining $\sigma^{2}$ is given by the equation ${ }^{3,4}$

$$
\begin{aligned}
\sigma^{2} \int_{0}^{a} e^{(3 \lambda-\nu) / 2}(P+E) r^{2} \xi^{2} d r= & 4 \int_{0}^{a} e^{(\lambda+\nu) / 2} r P^{\prime} \xi^{2} d r \\
& +\int_{0}^{a} e^{(\lambda+3 \nu) / 2}\left[\gamma P / r^{2}\right]\left(r^{2} e^{-\nu / 2} \xi\right)^{\prime 2} d r \\
& -\int_{0}^{a} e^{(\lambda+\nu / 2)}\left[P^{\prime 2} /(P+E)\right] r^{2} \xi^{2} d r \\
& +8 \pi \int_{0}^{a} e^{(3 \lambda+\nu) / 2} P(P+E) r^{2} \xi^{2} d r
\end{aligned}
$$


A sufficient condition for the dynamical stability of a mass is that the righthand side of Eq. (2) vanishes for some chosen "trial function" $\xi$ which satisfies the boundary conditions

$$
\xi=0 \quad \text { at } \quad r=0,
$$

and

$$
\begin{aligned}
\delta P & =-\xi P^{\prime}-\gamma P e^{\nu / 2}\left[\left(r^{2} e^{-\nu / 2} \xi\right)^{\prime} / r^{2}\right] \\
& =0 \quad \text { at } \quad r=a,
\end{aligned}
$$

where $a$ is the size of the configuration, $\delta P$ is the 'Lagrangian displacement in pressure' and the prime denotes radial derivative. The quantity $\gamma$ is defined as ${ }^{3,4}$

$$
\gamma=[(P+E) / P](\Delta P / \Delta E)
$$

where $\Delta P$ and $\Delta E$ denote the 'Eulerian change' in pressure and energy density, respectively. With this definition of $\gamma$, the boundary conditions [Eqs. (3) and (4)] become

$$
\xi=0 \quad \text { at } \quad r=0,
$$

and

$$
\Delta P=-\gamma P e^{\nu / 2}\left[\left(r^{2} e^{-\nu / 2} \xi\right)^{\prime} / r^{2}\right]=0 \quad \text { at } \quad r=a .
$$

For an adiabatic perturbation, using the relation ${ }^{11-13}$

$$
\frac{\Delta P}{\Delta E}=(\partial P / \partial E)_{s},
$$

where $s$ is the specific entropy, and if $n$ denotes the number density, such that, $P \equiv P(E, n)$, Eq. (5) becomes

$$
\gamma=(n / P)(\partial P / \partial n)_{s}=[(P+E) / P](\partial P / \partial E)_{s},
$$

or,

$$
v^{2}=\gamma P /(P+E)=\text { Finite (as long as } \gamma \text { is finite). }
$$

Let us consider the homogeneous sphere of uniform energy density, $E$. The equations governing equilibrium ${ }^{3,4,14}$ can be written in the form of compaction parameter $u$ and the radial co-ordinate measured in units of configuration size $y(\equiv r / a)$ as

$$
\begin{aligned}
8 \pi E a^{2} & =6 u, \\
8 \pi P a^{2} & =6 u \frac{\left(1-2 u y^{2}\right)^{1 / 2}-(1-2 u)^{1 / 2}}{3(1-2 u)^{1 / 2}-\left(1-2 u y^{2}\right)^{1 / 2}}, \\
e^{-\lambda} & =\left(1-2 u y^{2}\right), \\
e^{\nu} & =(1 / 4)\left[3(1-2 u)^{1 / 2}-\left(1-2 u y^{2}\right)^{1 / 2}\right]^{2} .
\end{aligned}
$$


Table 1. Various values of the compaction parameter $u$ and the corresponding critical (minimum) values of $\gamma\left(\gamma_{\text {crit }}\right)$ compatible with dynamical stability of compressible homogeneous sphere of uniform energy density, $E$. $(P / E)_{0}$ and $v_{0}$ represent, respectively, the pressure-density ratio and the speed of sound at the center of the configuration. It is seen that the dynamical stability of causal configurations (i. e., $u \leq 0.340555$ ) is constrained by the 'ratio of the specific heats', $\gamma \leq 2.594570$.

\begin{tabular}{cccc}
\hline$u$ & $\gamma_{\text {crit }}$ & $(P / E)_{0}$ & $v_{0}^{2}$ \\
\hline 0.050000 & 1.38400 & 0.02780 & 0.03743 \\
0.100000 & 1.44910 & 0.06272 & 0.08552 \\
0.150000 & 1.53550 & 0.10817 & 0.14990 \\
0.200000 & 1.65620 & 0.17027 & 0.24097 \\
0.250000 & 1.83750 & 0.26120 & 0.38056 \\
0.277800 & 1.98430 & 0.33340 & 0.49615 \\
0.300000 & 2.14110 & 0.40958 & 0.62210 \\
0.320000 & 2.32890 & 0.50000 & 0.77630 \\
0.333300 & 2.49000 & 0.57710 & 0.91118 \\
0.340555 & 2.59457 & 0.62710 & 1.00000 \\
\hline
\end{tabular}

Equation (2) is evaluated for Eqs. (11) with respect to the trial function, $\xi=r e^{\nu / 4}$ [because it gives the most rigorous results ${ }^{15,16}$ among the various trial functions of the form $\xi=r e^{\nu / N}, N=2,3,4 \ldots \infty$, and the form given as $\xi=b_{1} r\left(1+a_{1} r^{2}+a_{2} r^{4}+a_{3} r^{6}+\ldots\right) e^{\nu / 2}$, where $a_{1}, a_{2}, a_{3}, \ldots$ are adjustable constants $^{17,27}$ ] for various assigned values of the constant $\gamma$, so that the configuration becomes compressible, and the speed of sound in this medium remains finite and is given by [from Eqs. (10) and (11)]

$$
v^{2}=\gamma\left[1-\exp \left(\nu-\nu_{a}\right],\right.
$$

where the subscript ' $a$ ' denotes the value of the corresponding quantity at the surface of the configuration.

The compaction parameters of dynamically stable configurations compatible with causality [i.e., $v \leq 1$ ] are given in Table 1 . These values of $\gamma$ are consistent with those obtained by Chandrasekhar. ${ }^{3,4}$ It is seen that the compactness of the causal configuration, $u \leq 0.34056$ is constrained by the 'ratio of the specific heats, $\gamma \leq 2.59457$. Notice that for a perfectly incompressible homogeneous fluid sphere of uniform energy density, $E=n$, and the ratio of the specific heats, $\gamma$, and the speed of sound in this medium become infinity for all values of $u \leq(4 / 9)$, and the configuration would be dynamically stable for all values of $u \leq(4 / 9)$.

\section{Application to Obtain an Absolute Upper Bound on Mass and Uniform Rotation of Relativistic Stars}

By assigning the energy density $E$ equal to the average nuclear density, i.e. $E=$ $2 \times 10^{14} \mathrm{~g} \mathrm{~cm}^{-3},{ }^{7}$ in Eqs. (11) for the compaction parameter, $u=0.34056$, we obtain 
a maximum mass of $5.4 M_{\odot}$ [which is larger than the masses obtained earlier]. ${ }^{7-9}$

Although, the assigned value of energy density thus substituted is reasonable, it might be fiduciary. Therefore, we have to constrain the value of the energy density by some observational fact, and it may be constrained by the fastest rotating pulsar, PSR $1937+20$, with rotation period, $P=1.558 \mathrm{~ms}$, known to date. ${ }^{18}$

The determination of maximum masses of neutron stars corresponding to maximum rotation rates require complete general relativistic calculations. ${ }^{19,20}$ However, the value of $E$ and hence the maximum mass of a static (non-rotating) configuration, corresponding to higher $u$ values, can be obtained very accurately (see, e. g. Refs. 21 and 22) by using the empirical formula given by Koranda, Stergioulas, and Friedman ${ }^{23}$ in the following form

$$
P_{\text {rot }, \min }(\mathrm{ms})=0.740\left[M_{\max } / M_{\odot}\right]^{-1 / 2}\left[a_{\max } / 10 \mathrm{~km}\right]^{3 / 2},
$$

where $P_{\text {rot,min }}$ is the (minimum) rotation period corresponding to a configuration, rotating (uniformly) with maximum angular velocity, and $M_{\max }$ and $a_{\max }$ represent, respectively, the maximum mass and the corresponding size of the non-rotating configuration. Rewriting Eq. (13) in terms of compaction parameter, $u[\equiv M / a]$, and angular velocity $\Omega_{\max }\left(\equiv 2 \pi / P_{\text {rot }, \min }\right)$ we obtain

$$
\Omega_{\max }=2.21 \times 10^{10}\left[u_{\max }^{1 / 2} / a_{\max }(\mathrm{cm})\right] \mathrm{s}^{-1}
$$

where $u_{\max }$ is the maximum $u$ value of the non-rotating configuration, such that the configuration becomes dynamically unstable when $u$ exceeds $u_{\max }$, and $a_{\max }$ represents the corresponding radius of the configuration. Note that the corresponding formula which gives an error of $4-5 \%$ was previously obtained by Haensel and Zdunik. ${ }^{24}$ By the use of Eqs. (11) into Eq. (14), we obtain

$$
E\left(\mathrm{~g} \mathrm{~cm}^{-3}\right)=6.59 \times 10^{6}\left[\Omega_{\max }\left(\mathrm{s}^{-1}\right)\right]^{2},
$$

and,

$$
M_{\max }(\mathrm{cm})=\left[3 u_{\max }^{3} / 4 \pi E\left(\mathrm{~cm}^{-2}\right)\right]^{1 / 2} .
$$

Thus, the uniform energy density of the configuration depends only upon the rotation period, and not upon the compaction parameter $u$. Therefore, it is clear from Eqs. (15) and (16) that for a given value of the rotation period $P_{\text {rot }}$ the maximum mass of the stable configuration depends only upon the maximum value of $u\left(u_{\max }\right)$. For $P_{\text {rot }}=1.558 \mathrm{~ms}$, Eq. (15) gives the energy density, $E$, of the configuration as $1.072 \times 10^{14} \mathrm{~g} \mathrm{~cm}^{-3}$, the substitution of $u_{\text {max }} \cong 0.34056$ from Table 1 into Eq. (16) gives the maximum mass of the configuration, $M_{\max } \cong 7.387 M_{\odot}$ and the corresponding radius, $a_{\max } \cong 31.974 \mathrm{~km}$. Notice that the range of energy density obtained in this manner is applicable to the baryonic equation of state, known as $Q$-Star equation of state. ${ }^{5,22,25}$

For the value of $u \cong 0.34056$, the speed of sound is maximum, $v \cong 1$, at the center and decreases monotonically from center to the surface of the configuration, and at the surface it vanishes along with the pressure. These masses have important 
implications regarding massive compact objects like Cyg X-1, Cyg XR-1, and LMC $\mathrm{X}-3$ etc. These limits of maximum masses could be updated if the faster rotating pulsars are observed in the future [see, Ref. 22 for a detailed discussion in this regard].

On the other hand, if we impose the constraint on energy density [by using Eq. (16)] such that the maximum mass of neutron star is $1.442 M_{\odot},{ }^{10}$ then Eq. (15) gives the minimum rotation period $0.3041 \mathrm{~ms}$ for the maximum $u \cong 0.34056$, and the corresponding uniform energy density $E$ is obtained as $2.813 \times 10^{15} \mathrm{~g} \mathrm{~cm}^{-3}$.

\section{Results and Conclusions}

An absolute upper bound on compaction parameter, $u \leq 0.34056$ [or the surface redshift $\leq 0.771]$, compatible with causality and the ratio of the specific heats, $\gamma \leq 2.59457$, is obtained by using the dynamically stable compressible homogeneous sphere. This upper limit of compaction parameter gives

(i) The maximum static mass of conventional model of neutron stars [taking $\left.E=2 \times 10^{14} \mathrm{~g} \mathrm{~cm}^{-3}\right]^{7,8}$ as $5.4 M_{\odot}$. This is greater than $4.8 M_{\odot}$ considered as an upper limit earlier.

(ii) The maximum mass of static neutron star exceeds to the value of $7.387 M_{\odot}$ which is greater than the upper limit of $5.3 M_{\odot}$ for neutron stars (so called $Q$-star models) obtained by Hochron, Lynn and Selipesky. ${ }^{26}$ This may have important implications for the heavy compact objects like Cyg X-1, Cyg XR-1, and LMC-X3 which may not, necessarily, be black holes.

(iii) The minimum rotation periods for a static $1.442 M_{\odot}$ neutron star to be $0.3041 \mathrm{~ms}$ with a uniform energy density $E$ as $2.813 \times 10^{15} \mathrm{~g} \mathrm{~cm}^{-3}$.

(iv) A causally consistent and dynamically stable model of ultra-compact objects

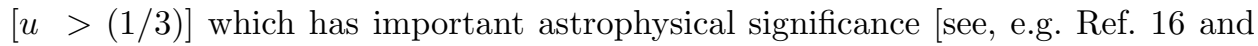
references therein].

\section{Acknowledgments}

The author acknowledges State Observatory, Nainital for providing library and computer center facilities.

\section{References}

1. K. Schwarzschild, Sitzer. (Preuss. Akad. Wiss. Berlin, 1916) p. 424.

2. H. A. Buchdahl, Phys. Rev. 116, 1027 (1959).

3. S. Chandrasekhar, Phys. Rev. Lett. 12, 114 \& 437 (1964a).

4. S. Chandrasekhar, Astrophys. J. 140, 417 (1964b).

5. J. F. Dolan, Astrophys. J. 384, 249 (1992).

6. W. D. Arnett and R. L. Bowers, Astrophys. J. Suppl. 33, 415 (1977).

7. K. Brecher and G. Caporaso, Nature 259, 377 (1976).

8. J. B. Hartle, Phys. Rep. 46, 201 (1978).

9. C. E. Rhoades Jr. and R. Ruffini, Phys. Rev. Lett. 32, 324 (1974). 
10. J. H. Taylor and J. M. Weisberge, Astrophys. J. 345, 434 (1989).

11. J. H. Jeans, Astronomy and Cosmology (London: Cambridge Univ. Press, 1928).

12. M. Merafina and R. Ruffini, Astron. ES Astrophys. 221, 4 (1989).

13. M. Merafina and R. Ruffini, Astron. ES Astrophys. 227, 415 (1990).

14. R. C. Tolman, Phys. Rev. 55, 364 (1939).

15. P. S. Negi and M. C. Durgapal, Astrophys. \& Space Sci. 245, 97 (1996).

16. P. S. Negi and M. C. Durgapal, Gen. Rel. Grav. 31, 13 (1999).

17. J. M. Bardeen, K. S. Thorne and D. W. Meltzer, Astrophys. J. 145, 505 (1966).

18. D. C. Backer, S. R. Kulkarni, C. Heiles, M. M. Davis and W. M. Goss, Nature 300, 615 (1982).

19. J. L. Friedman, J. R. Ipser and L. Parker, Astrophys. J. 304, 115 (1986).

20. J. L. Friedman, J. R. Ipser and L. Parker, Phys. Rev. Lett. 62, 3015 (1989).

21. P. S. Negi and M. C. Durgapal, Astron. ES Astrophys. 353, 641 (2000).

22. P. S. Negi and M. C. Durgapal, Astrophys. ES Space Sci. 275, 299 (2001).

23. S. Koranda, N. Stergioulas and J. L. Friedman, Astrophys. J. 488, 799 (1997).

24. P. Haensel and J. L. Zdunik, Nature 340, 617 (1989).

25. S. Bahcall, B. W. Lynn and S. B. Selipesky, Astrophys. J. 362, 251 (1990).

26. D. A. Hochron, B. W. Lynn and S. B. Selipesky, Class. Quantum Grav 10, 299 (1993).

27. R. F. Tooper, Astrophys. J. 142, 1541 (1965). 